\title{
Effects of lipid composition on membrane permeation ${ }^{\dagger}$
}

\author{
Michail Palaiokostas, ${ }^{a}$ Wei Ding, ${ }^{a}$ Ganesh Shahane, ${ }^{a}$ and Mario Orsi ${ }^{b *}$
}

Passive permeation through lipid membranes is an essential process in biology. In vivo membranes typically consist of mixtures of lamellar and nonlamellar lipids. Lamellar lipids are characterized by their tendency to form lamellar sheet-like structures, which are predominant in nature. Nonlamellar lipids, when isolated, instead form more geometrically complex nonlamellar phases. While mixed lamellar/nonlamellar lipid membranes tend to adopt the ubiquitous lamellar bilayer structure, the presence of nonlamellar lipids is known to have profound effects on key membrane properties, such as internal distributions of stress and elastic properties, which in turn may alter related biological processes. This work focuses on one such process, i.e., permeation, by utilising atomistic molecular dynamics simulations in order to obtain transfer free energy profiles, diffusion profiles and permeation coefficients for a series of thirteen small molecules and drugs. Each permeant is tested on two bilayer membranes of different lipid composition, i.e., purely lamellar and mixed lamellar/nonlamellar. Our results indicate that the presence of nonlamellar lipids reduces permeation for smaller molecules (molecular weight $<100$ ) but facilitates it for the largest ones (molecular weight $>100$ ). This work represents an advancement towards the development of more realistic in silico permeability assays, which may have a substantial future impact in the area of rational drug design.

\section{Introduction}

Biological membranes are fundamental structures responsible for the encapsulation of cells, as well as the compartmentalisation of their content. The core of any biological membrane is the lipid bilayer, which in vivo is composed of up to hundreds of different types of lipid molecules. Membrane lipids are amphiphilic molecules that are typically characterised by two parts; a polar head group, and a non-polar tail comprising esterified fatty acids. Lipid molecules vary widely in terms of size, chemical structure and polarity. Due to many possible combinations of lipid molecules, lipid assemblies can exhibit a wide variety of physical properties and structures ${ }^{1,2}$.

In particular, it is possible to distinguish lipid phases that are 'lamellar' or 'nonlamellar', corresponding to two fundamental structures that are radically different from each other. The lamellar phase, ubiquitous in biology, is characterized by the typical bilayer arrangement, whereby the hydrophilic heads are exposed to bulk aqueous environments while the hydrophobic tails assemble into the membrane inner core, overall forming twodimensional sheet-like structures that are widespread in nature. Nonlamellar phases form instead assemblies of very different and

\footnotetext{
a School of Engineering and Materials Science, Queen Mary University of London, London, United Kingdom

${ }^{b}$ Department of Applied Sciences, University of the West of England, Bristol, United Kingdom; E-mail: mario.orsi@uwe.ac.uk

* Corresponding author

$\dagger$ Electronic Supplementary Information (ESI) available: Permeants' properties, convergence of simulations, diffusion coefficients analysis, published permeation coefficients, hypothesis tests, lateral mobility. See DOI: 10.1039/b000000x/
}

more complex geometries, depending on the specific lipid types and thermodynamic conditions, such as inverse hexagonal structures characterized by lipid-lined water channels. Nonlamellar phases are uncommon, and typically only appear in transient processes such as membrane fusion. Notably, in vivo cell membranes are mixtures comprising both lamellar and nonlamellar lipids, even though the overall structures formed are almonst invariably lamellar $^{2-5}$. In this study, we consider the representative lamellar lipid dioleoylglycerophosphocholine (DOPC) and the representative nonlamellar lipid dioleyolglycerophosphoethanolamine (DOPE), whose chemical structures are reported in figure 1. Under biological conditions, when dispersed in an aqueous environment, pure DOPC forms lamellar phases, while pure DOPE forms nonlamellar (inverse hexagonal) phases ${ }^{6}$. Structurally, DOPC and DOPE are identical apart from the head terminal group, which is choline for DOPC and ethanolamine for DOPE (see figure 1). This difference in the aminoalcohol affects the size and shape of the polar head, making DOPC lipids bulkier.

The effect of different lipids on the mechanical properties and dynamic behaviour of bilayer membranes has been studied extensively in the past using experiments ${ }^{7,8}$, analytical theory ${ }^{9,10}$ and molecular dynamics (MD) simulations ${ }^{11-22}$. In particular, Ding et al. ${ }^{21}$ have employed atomistic simulations to quantify the effects of changes in the ratio of lamellar vs. nonlamellar lipids on the physical properties of bilayer membranes. A key finding was that the addition of DOPE lipids to DOPC bilayers had a negligible effect on the structure of the bilayers but induced substantial changes to the lateral pressure profile, in agreement with the available qualitative experimental evidence ${ }^{7}$. The 


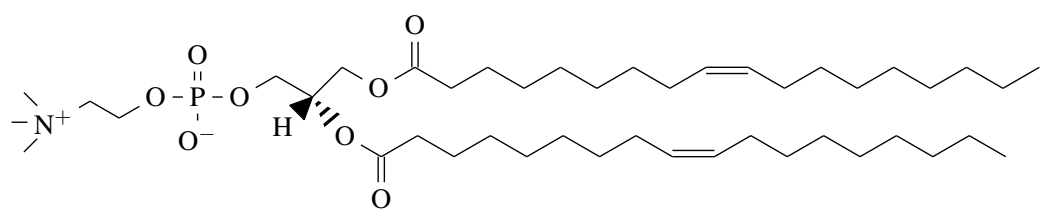

(a) 1,2-dioleoyl-sn-glycero-3-phosphocholine (DOPC)

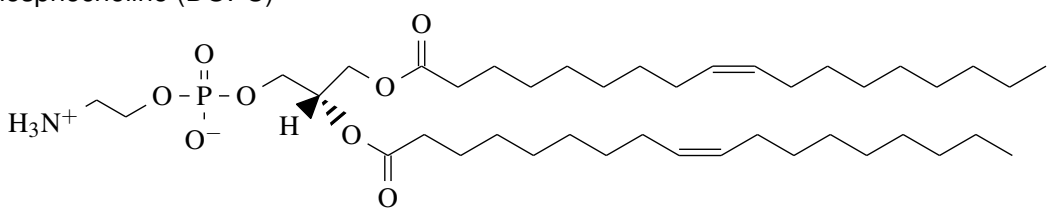

(b) 1,2-dioleoyl-sn-glycero-3-phosphoethanolamine (DOPE)

Fig. 1 Skeletal chemical structures of the phospholipids simulated in this study.

lateral pressure profile, arguably the most fundamental physical property of lipid bilayers ${ }^{2}$, characterizes the net stresses across membranes, thus quantifying the pressure that membrane inclusions (such as proteins) or permeants "feel" inside the bilayer ${ }^{23}$. This pressure varies over a vast range (hundreds of atmospheres) with the bilayer depth, and three distinct regions can be identified depending on the nature of the forces present; a region of high repulsion corresponding to the upper lipid headgroups, a region of high attraction in the polar/apolar interface, and a region of varying repulsion in the bilayer hydrophobic core. The lateral pressure profile underlies many fundamental membrane phenomena, such as phase transition ${ }^{24,25}$, water penetration ${ }^{26}$, drug transport ${ }^{27,28}$, anaesthesia ${ }^{29-32}$ and in general membrane protein function ${ }^{23,33-38}$. For instance, changes in the lateral pressure profile are believed to control the opening and closing of ion channels ${ }^{33}$.

Spontaneous passive permeation, a fundamental transport mechanism across lipid membranes, is driven by the concentration gradient of the permeating molecule between the extracellular fluid and the cytoplasm ${ }^{39,40}$. Uncharged small molecules and drugs permeate passively through lipid membranes ${ }^{41,42}$, therefore understanding the molecular mechanism of passive permeation is of immense importance for drug design and drug delivery applications. Past permeation studies can be distinguished in two categories: those that examine the effect of permeants' characteristics and those that examine the effect of lipid molecules. For the former, common parameters are the size ${ }^{43-46}$, shape 44,47 , solubility $^{47-49}$ and charge $\mathrm{e}^{50,51}$. For the latter, published research examined the effect of the length of lipid chains ${ }^{15,52-55}$, unsaturation $^{56-59}$ and different headgroups ${ }^{60-62}$. Also, many studies have focused on the addition of cholesterol ${ }^{48,62-69}$.

With regards to lamellar/nonlamellar mixtures, we are aware of only two relevant previous studies, both experimental. Huster et al. ${ }^{56}$ observed that the addition of nonlamellar DOPE lipids reduced the permeation of water by $40 \%$ in comparison to a pure DOPC bilayer and by $18 \%$ when mixed with a pure polyunsaturated 18:1-22:6 PC bilayer. The results were explained based on lipid order increase, which is more prominent in saturated and monounsaturated lipids, leading to tighter packing and smaller area per lipid. Purushothaman et al. ${ }^{70}$ examined the permeation of the antibiotic norfloxacin through pure and mixed DOPC,
DOPE and DOPG bilayers. The addition of DOPE lipids generally reduced the permeation coefficient, although the reduction was higher for smaller concentrations of DOPE.

In this work, we use molecular dynamics simulations to characterize the effects of a change in the lamellar/nonlamellar lipid composition on passive permeation by simulating bilayers comprising either pure DOPC (lamellar) or a 1 to 3 mixture of DOPC to DOPE (nonlamellar) lipid molecules, respectively. Bilayers comprising different relative concentrations of these two lipid were shown previously to differ substantially in their lateral pressure profile $7,21,71$. In particular, an increase in repulsive forces was observed in the hydrophobic part of the bilayer when DOPE (nonlamellar) lipids were present. We hypothesise that the increase in repulsive forces should lead to an increase in the resistance to permeation through the membrane. In the remainder of the paper, we report a series of results produced with atomistic molecular dynamics simulations for 13 small molecules and drugs, namely ammonia, water, fluoromethane, carbon dioxide, propane, ethanol, urea, isopropanol, glycine, phenol, benzoic acid, coumarin and paracetamol. The chemical structure of all permeants is shown in figure 2 while their physical properties are reported in the supplementary material (table S1 ).

\section{Methodology}

\subsection{Permeability calculations}

A simple framework to predict membrane permeability, first introduced over a hundred years ago by Meyer and Overton 72,73, is based on the octanol-water partition coefficient $\log P_{\text {oct/water }}$ of a permeant, which indicates the permeant's solubility preference between an octanol and a water phase. On this basis, the bulk solubility-diffusion model of permeability was proposed ${ }^{74}$ in which lipid membranes were considered as homogeneous bulk bodies. Diamond et al. ${ }^{75}$ later accounted for the heterogeneity of membranes by developing the inhomogeneous solubilitydiffusion model, whereby the permeation coefficient $P$ of a solute through a membrane can be predicted as ${ }^{76,77}$ :

$$
P=1 / \int_{z_{1}}^{z_{2}} R(z) d z=1 / \int_{z_{1}}^{z_{2}} \exp \left[\frac{\Delta G(z)}{\mathrm{R} T}\right] \frac{1}{D(z)} d z
$$


$\mathrm{NH}_{3}$

(a) Ammonia

$$
\mathrm{O}=\mathrm{C}=\mathrm{O}
$$

(d) Carbon dioxide<smiles>NC(N)=O</smiles>

(g) Urea

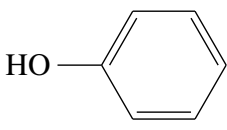

(j) Phenol
$\mathrm{H}_{2} \mathrm{O}$

(b) Water<smiles>CCC</smiles>

(e) Propane<smiles>CC(C)O</smiles>

(h) Isopropanol

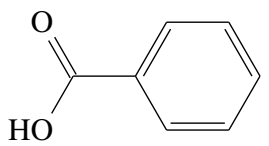

(k) Benzoic Acid (c) Fluoromethane<smiles>CCO</smiles>

(f) Ethanol<smiles>NCC(=O)O</smiles>

(i) Glycine

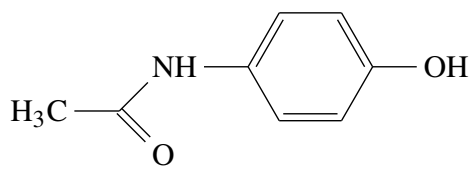

(m) Paracetamol

Fig. 2 Skeletal chemical structures of the permeants simulated in this study.

where $T$ is the simulation temperature, $\mathrm{R}$ is the universal gas constant (which is equal to the product of Boltzmann's constant $k_{B}$ with Avogadro's number $\mathrm{N}_{\mathrm{A}}, \mathrm{R}=\mathrm{k}_{\mathrm{B}} \cdot \mathrm{N}_{\mathrm{A}}$ ) and $z$ is the position normal to the bilayer surface, with $z_{1}$ and $z_{2}$ representing the bulk water regions on the two sides of the membrane. Also, $R(z)$ is the local resistance to permeation, $D(z)$ is the local diffusion coefficient of the solute and $\Delta G(z)$ is the Gibbs free energy difference between the thermodynamic states of the permeant in bulk water and at position $z$. To obtain $\Delta G(z)$ and $D(z)$ from molecular dynamics simulations, several enhanced sampling approaches have been developed, such as the z-constraint or the z-restraint methods, which are discussed in detail elsewhere ${ }^{78-80}$. In this work we utilise the z-restraint method.

The free energy difference can be obtained by using the umbrella sampling scheme ${ }^{81}$, whereby a harmonic potential restrains the movement of the permeant in a small "window" around each position along the reaction coordinate path, which is the z-direction normal to the bilayer plane in our work. The free energy difference is then calculated as:

$$
\Delta G(z)=-\mathrm{R} T \ln P_{b}(z)+V_{b}(z)
$$

where $V_{b}(z)$ is the biasing potential and $P_{b}(z)$ is the permeant's spatial distribution along $\mathrm{z}$ positions. Finally, in order to obtain the unbiased free energy difference $\Delta G$, the weighted histogram analysis method (WHAM) is used ${ }^{82,83}$.

Regarding the diffusion coefficient $(D(z)$ in equation 1$)$, it should be noted that, in general, computing reliable local diffusion coefficients from restrained simulations remains a very active research field ${ }^{84-87}$. One of the most popular methods is the one introduced by Hummer ${ }^{88}$ (in turn based on the previous works of Woolf and Roux ${ }^{89}$ and Berne et al. ${ }^{90}$ ). In this method, when umbrella sampling simulations are performed with a harmonic bias along a reaction coordinate, the diffusion coefficient can be computed as:

$$
D(z)=\frac{\operatorname{var}(z)}{\tau}
$$

where $\operatorname{var}(z)=\left\langle z^{2}\right\rangle-\langle z\rangle^{2}$ is the variance of the $\mathrm{z}$-distance between the centres-of-mass of the permeant and membrane and $\tau$ is the characteristic time of the z-distance autocorrelation function:

$$
\tau=\int_{0}^{\infty} \frac{\langle\delta z(t) \delta z(0)\rangle}{\operatorname{var}(z)} d t
$$

where according to the definition of the autocorrelation function $\delta z(t)=z(t)-\langle z\rangle$. The integral can be computed with the method proposed by O'Neill et al. ${ }^{91}$, where the integration domain is taken from the beginning until the first time that the autocorrelation function becomes zero. Finally, permeability coefficients are computed by direct substitution into equation 1 .

It must be noted that permeation through water pores has also been proposed as an alternative or complimentary pathway to the solubility-diffusion mechanism. ${ }^{92}$ In particular, the importance of pore mediated permeation has been recently established for large, charged molecules such as cell penetrating peptides. ${ }^{93,94}$ However, for smaller and neutral molecules, such as those considered in this work, the solubility-diffusion mechanism is regarded as predominant. ${ }^{95-97}$ 


\subsection{Simulation Protocol}

Each simulation conducted in this study comprised 4300 water molecules, 128 lipid molecules (64 per leaflet) and 1 permeating molecule (see figure 2 for the full set of permeants considered). Each permeant was simulated in two membrane systems, pure (comprising DOPC only) and mixed (DOPC:DOPE (1:3) mixture). A typical snapshot for the mixed system is reported in figure 3. Both starting configurations for the membrane systems

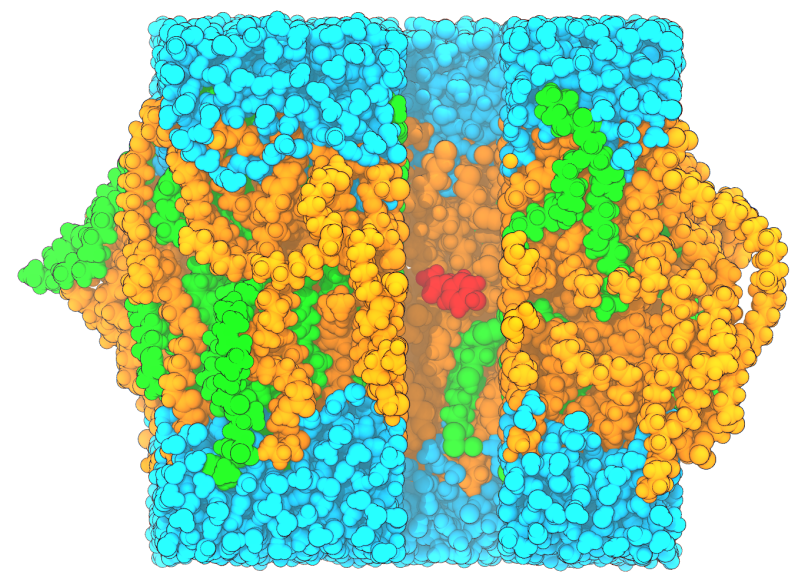

Fig. 3 Representative simulation snapshot of a DOPC:DOPE(1:3) bilayer with the permeant paracetamol. Water molecules are cyan, DOPC lipids are green, DOPE lipid are orange, and the permeant is red (for clarity, nonpermeant molecules were removed from a cylindrical region encompassing the permeant).

were taken pre-equilibrated from our previous work ${ }^{21}$, and the permeant was manually placed in the required position along the bilayer. Initially, an energy minimisation was performed to remove any high energy overlaps and then a short constant temperature and pressure (NPT) 100 ps equilibration was run. Both during the minimisation and the equilibration, the distance between the centres of mass of the membrane and the permeant was constrained, in order to ensure the correct distance between them in the beginning of the production simulation. Overall, 28 z-positions were examined for each permeant-membrane combination, and for each such case a $100 \mathrm{~ns}$ NPT production simulation was performed. Molecular dynamics simulations were conducted with the GROMACS software ${ }^{98-103}$ version 5.1.1. Van der Waals forces were approximated with a Lennard-Jones potential with a switching cutoff from $1 \mathrm{~nm}$ to $1.2 \mathrm{~nm}$, short range electrostatics were approximated with a Coulomb potential with a cutoff at $1.2 \mathrm{~nm}$ and long range electrostatics were treated with the Smooth Particle-Mesh Ewald (SPME) ${ }^{104}$ method. Lipid molecules were modelled with the CHARMM36 (August 2015 version) force field ${ }^{105,106}$, permeant molecules were modelled with CHARMM36 or compatible CGenFF ${ }^{107,108}$ parameters and water molecules were modelled with the CHARMM implementation of TIP3P ${ }^{109}$.

For temperature coupling during the equilibration and production simulations, the V-rescale algorithm ${ }^{110}$ was used. All the systems were kept at $300 \mathrm{~K}$ and the coupling time constant was set to $1 \mathrm{ps}$. Pressure was kept at 1 bar with the Berendsen ${ }^{111}$ and Parrinello-Rahman ${ }^{112-114}$ barostats during equilibration and production simulations, respectively; the coupling time constant was $5 \mathrm{ps}$ and the coupling type was semi-isotropic, i.e., isotropic for the $\mathrm{x}$ and $\mathrm{y}$ directions but independently from the $\mathrm{z}$ direction (as is common practice in simulations of interfacial systems $\left.{ }^{34,94,115}\right)$. A 2 fs timestep was used; covalent bonds with hydrogen atoms were constrained with the SETTLE algorithm ${ }^{116}$ for water molecules and with the LINCS algorithm ${ }^{117,118}$ for all other molecules.

\subsection{Analysis}

The restraining forces and z-axis fluctuations of the permeant were sampled every $1 \mathrm{ps}$ resulting in two timeseries of $10^{5}$ points each. The first $30 \mathrm{~ns}$ (30000 points) were discarded as extra equilibration time and all final results were produced using the last 70 ns. Standard errors for the calculated properties were computed with the block averaging method ${ }^{119,120}$, where the $70 \mathrm{~ns}$ timeseries were separated in 7 blocks of $10 \mathrm{~ns}$. In order to increase the computational efficiency and since the monolayers' composition was the same for each examined membrane, the permeants were positioned only across one leaflet of the bilayer. Afterwards, the position-dependent results were treated as symmetrical to cover the entire z-dimension of the bilayer.

The free energy profiles $\Delta G(z)$ were computed with the GROMACS implementation ${ }^{121}$ of the weighted histogram analysis method $^{83,122}$. Each permeant was restrained by a virtual spring with a harmonic force constant of $1000 \mathrm{~kJ} \mathrm{~mol}^{-1} \mathrm{~nm}^{-2}$ along the $\mathrm{z}$-axis, for 28 positions, every $0.1 \mathrm{~nm}$ from the water slab to the bilayer core. Local diffusion coefficients $D(z)$ were computed according to equation 3; extra analysis on the numerical integration of the autocorrelation function and the handling of oscillatory profiles with the application of filters is reported in the supplementary material. The resistance profiles and the permeability coefficients were computed by direct substitution of $\Delta G(z)$ and $D(z)$ into equation 1 . Statistical significance testing of the differences between permeation coefficients for the pure and mixed membranes was carried out with paired t-tests. Hydrogen bonds were computed between the permeants and the lipid-water molecules. The permeant lateral mobility was investigated qualitatively through the corresponding lateral trajectories; representative traces are reported in the supplementary information.

\section{Results and Discussion}

\subsection{Free-energy profiles}

The permeation free energy profiles $\Delta G(z)$ of the selected thirteen permeant molecules, simulated in both the pure DOPC and DOPC:DOPE(1:3) bilayers, are shown in the first column of figure 4.

The convergence study of the profiles is presented in detail in the supplementary material; while general features are analyzed as in previous studies, ${ }^{123,124}$ we report a novel approach to measure relative convergence.

The free energy difference represents the thermodynamic forces (entropic and enthalpic) that drive the process of perme- 

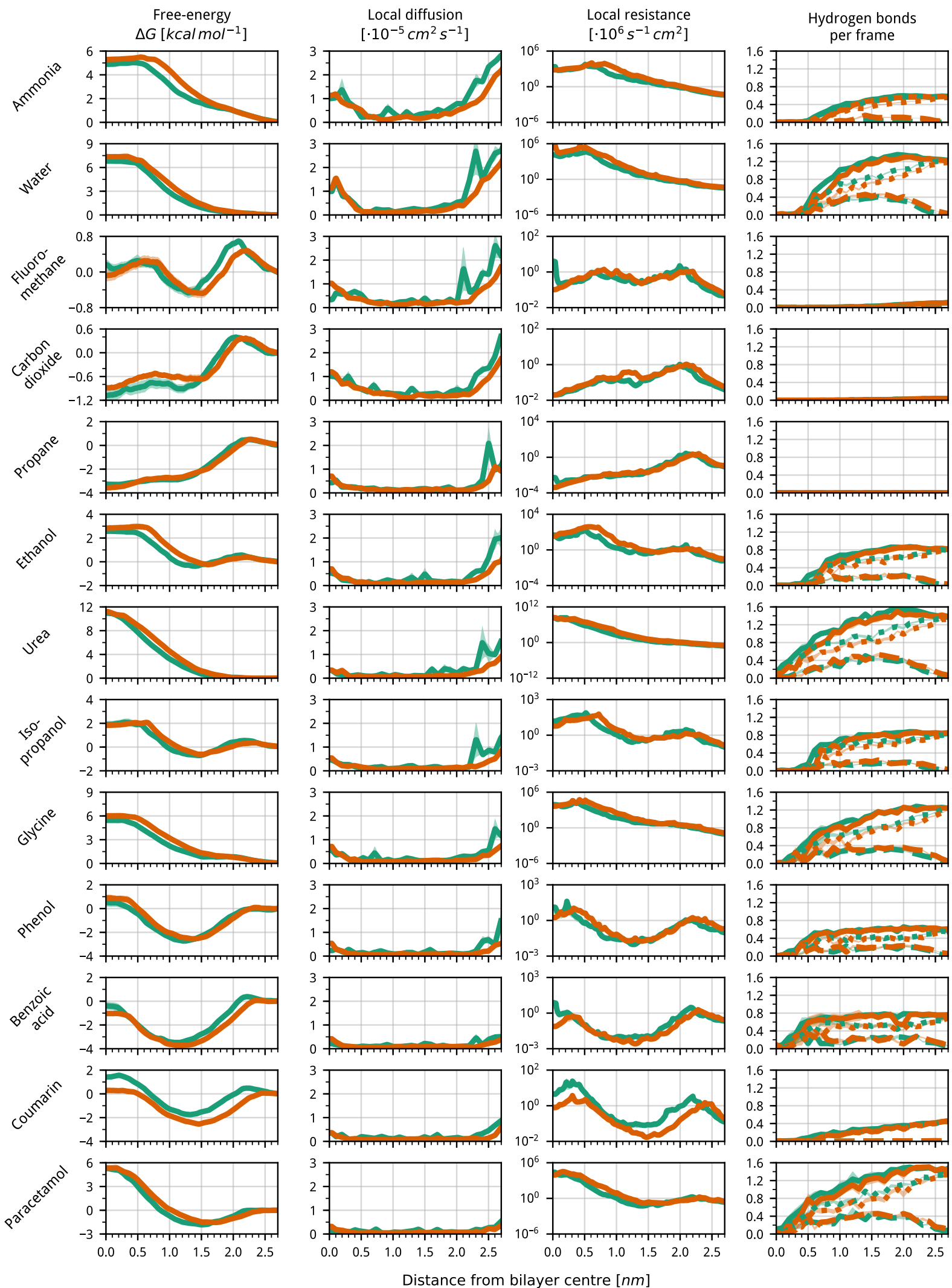

Distance from bilayer centre $[\mathrm{nm}]$

Fig. 4 Free-energy profiles (first column), local diffusion coefficients (second column), local resistance profiles (third column) and hydrogen bonds formed per frame (fourth column) for all the studied permeating molecules and membranes. The green lines correspond to the DOPC membrane and the orange lines correspond to the DOPC:DOPE(1:3) membrane. The standard error is represented with a semi-transparent area above and below the line of the average, although in most cases the standard error is smaller than the thickness of the line. For the hydrogen bonds results (fourth column), dashed lines represent hydrogen bonds formed between the permeants and lipid molecules, dotted lines represent hydrogen bonds formed between the permeants and water molecules and solid lines represent the sum of the two. 
ation, quantifying the spontaneity of the process and the preferred partitioning position of the permeant ${ }^{125}$. Considering our results, permeants can be separated into three distinct categories depending on where they exhibit the global minimum of their free energy difference, which predicts the location where the molecules preferentially partition.

In the first category, the free energy difference is always positive along the bilayer and the minimum position corresponds to the reference point for $\Delta G(z)=0$ in the water phase. This behaviour is observed for ammonia, glycine, urea and water; these molecules therefore are not predicted to partition inside the bilayer. Such a behaviour is consistent with the well-known polar hydrophilic nature of these compounds.

In the second category, the profiles have a small positive peak in the lipid head region, then exhibit a negative global minimum in the polar/apolar interface and finally a larger positive peak in the hydrophobic core. Coumarin for the DOPC membrane, ethanol and isopropanol, clearly belong to this category, with five more molecules exhibiting minor deviations from this behaviour. Benzoic acid has a peak in the hydrophobic core but is negative in value. Also, coumarin for the DOPC:DOPE(1:3) membrane, as well as paracetamol and phenol for both membranes, do not have a positive barrier in the head region. Fluoromethane has a positive peak in the head region, a global minimum in the interface, and a second positive peak close to the hydrophobic core; inside the tail region, the profile is marginally positive for the DOPC membrane, while marginally negative for the DOPC:DOPE(1:3) membrane. All these molecules have an amphiphatic nature and under physiological conditions they are known to partition in the polar/apolar interface ${ }^{126,127}$, therefore the free energy profiles are in agreement with the expected behaviour.

The third category includes carbon dioxide and propane, whose profiles feature a small positive peak in the lipid head region while being negative elsewhere, with global minima located in the bilayer centre, corresponding to their preferred partitioning position. Such behaviour is consistent with both permeants being known hydrophobic molecules.

In general, all free energy profiles presented here are in good qualitative agreement with previous studies of the same permeants and the same or different PC and PE lipids ${ }^{45-48,62,128-130 .}$ Furthermore, they all fit in the categories that were introduced by Neale et al. ${ }^{131}$ who classified over 200 free energy profiles of more than 100 small molecules from previous studies.

Permeants can also be classified by examining the effect of DOPE lipids on the transfer process. In the first group, comprising ammonia, glycine, urea and water, the free energy profile of the DOPC:DOPE mixture is the same or higher than the DOPC profile, across the whole bilayer depth. This is an indication that the presence of DOPE lipids increases the barrier to permeation, in parts or across the entire bilayer. For the second group, including carbon dioxide, ethanol, paracetamol and phenol, a lower DOPC:DOPE mixture profile is observed in the head group area but a higher DOPC:DOPE profile is observed in the interface and hydrophobic core area. Isopropanol and fluoromethane show a slight deviation from this behaviour by having a marginally lower peak in the DOPC:DOPE bilayer core than in the pure DOPC mem- brane. Benzoic acid, coumarin and propane form the third group, whereby the DOPC:DOPE mixture profile is the same or lower than the DOPC profile all across the bilayer. A summary of the permeants classification in relation to their free energy difference profiles is reported in Table 1.

Table 1 Permeants classification based on their free energy profile. Subscripts "PC" refers to DOPC and "PE" to DOPE.

\begin{tabular}{|c|c|c|c|}
\hline \multirow[b]{2}{*}{$\Delta G$ relations } & \multicolumn{3}{|c|}{ Partitioning area } \\
\hline & Bulk water & Lipid heads & Lipid tails \\
\hline$\Delta G_{\mathrm{PC}: \mathrm{PE}} \geq \Delta G_{\mathrm{PC}}$ & $\begin{array}{l}\text { Ammonia } \\
\text { Glycine } \\
\text { Urea } \\
\text { Water }\end{array}$ & & \\
\hline$\Delta G_{\mathrm{PC}}^{\text {tails }} \leq \Delta G_{\mathrm{PC}: \mathrm{PE}} \leq \Delta G_{\mathrm{PC}}^{\text {heads }}$ & & $\begin{array}{l}\text { Ethanol } \\
\text { Fluoromethane }^{* \dagger} \\
\text { Isopropanol }^{\dagger} \\
\text { Paracetamol }^{*} \\
\text { Phenol }^{*}\end{array}$ & Carbon dioxide \\
\hline$\Delta G_{\mathrm{PC}: \mathrm{PE}} \leq \Delta G_{\mathrm{PC}}$ & & $\begin{array}{l}\text { Benzoic acid* } \\
\text { Coumarin* }\end{array}$ & Propane \\
\hline
\end{tabular}

\subsection{Local diffusion profiles}

The local diffusion coefficients for all the permeants and membranes investigated are reported in the second column of figure 4. All cases exhibit the same qualitative behaviour; diffusion is largest in the water region, then it drops quickly as permeants approach the hydrophobic lipid tails, and finally increases again in the bilayer centre. Quantitatively, for both membranes, diffusion of heavier molecules is considerably slower, especially in the water region; for example, paracetamol has ten times slower diffusion in water than ammonia. With regards to the effect of DOPE lipids, there are no significant differences between the two examined membranes. For benzoic acid, fluoromethane, paracetamol and phenol, the peak in the bilayer core is marginally higher for the DOPC:DOPE mixture. Also, for all molecules, the diffusion profiles for the DOPC systems tend to be slightly higher than DOPC:DOPE profiles, especially in the water and headgroup regions. It can also be seen that some profiles exhibit pronounced fluctuations, especially in the water and headgroup regions; extra analysis of these effects is reported in the supplementary material. Overall, while diffusion coefficients are inherently noisy, it should be stressed that their contribution to the overall permeation model is marginal compared to the free energy term, which appears as argument of an exponential function (equation 1).

\subsection{Local resistance profiles}

The resistance profiles for both membrane systems and all permeants studied are reported in the third column of figure 4. Carbon dioxide and propane, both hydrophobic molecules, experience higher permeation resistance in the hydrophilic region of the 
bilayer, while hydrophilic molecules (ammonia, glycine, urea and water) experience the highest resistance in the bilayer core, as expected. Amphiphilic molecules are characterised by two peaks in their resistance profiles. The first features in the hydrophilic area of the bilayer, indicating a permeation barrier of the hydrophobic part of the permeant as it dissolves inside the hydrophilic region of the bilayer. The second can be seen in the lower chains region and the bilayer core, indicating a resistance to permeation of the hydrophilic part of the permeant in the hydrophobic region of the bilayer. The effect of the lipid composition depends on the hydrophilicity of the permeants. The DOPC:DOPE (1:3) bilayer induces higher resistance for hydrophilic permeants than the pure DOPC bilayer, especially in the lipid chains region. For hydrophobic molecules the resistance is marginally higher in the polar region of the DOPC:DOPE (1:3) bilayer but it is unaffected elsewhere. Finally, for the amphiphilic permeants, the DOPC:DOPE (1:3) membrane has lower resistance in the bilayer centre, higher resistance in the polar part and the $0.5 \mathrm{~nm}$ to $1.5 \mathrm{~nm}$ regions, and the same resistance in the other regions. Coumarin is an exception as the resistance is lower along the whole apolar part of the bilayer.

Overall it can be seen that, except for minor deviations, resistance profiles are qualitatively similar to the free energy profiles (compare first and third column of figure 4). Any deviation can be ascribed to differences in the diffusion behaviour, as reported also in previous permeation studies $46,47,132,133$. From our results, it can be seen that differences in the diffusion profiles can alter the relative resistance between the DOPC and DOPC:DOPE membrane for some permeants. In particular, resistance to permeation of both membranes to ammonia, urea, glycine and phenol becomes equal or higher for DOPC in the bilayer centre, although the free energy profile is clearly higher in the same region for the DOPC:DOPE mixed system. In other cases, e.g., for water, fluoromethane and benzoic acid, the differences between the membranes are amplified.

\subsection{Permeability}

Permeability coefficients for each molecule were computed from the local resistance profiles according to the inhomogeneous solubility-diffusion model as defined in equation 1 . The permeation values of the molecules examined are presented in ascending permeability order in table 2 along with selected representative literature results for the same permeants (an extended collection of all the available literature data for the examined molecules can be found in table S4 in the supplementary material).

The permeation coefficients of the examined molecules can be separated in two main groups when comparing between the two examined membranes. The first group includes the molecules for which the permeation coefficient is smaller corresponding to the DOPC:DOPE membrane than to the pure DOPC membrane, which are urea $(\approx-41 \%)$, water $(\approx-27 \%)$, glycine $(\approx-69 \%)$, ammonia $(\approx-71 \%)$, ethanol $(\approx-30 \%)$, isopropanol $(\approx-47 \%)$, phenol $(\approx-52 \%)$ and carbon dioxide $(\approx-30 \%)$. The second group includes the molecules with increased permeation through the DOPC:DOPE membrane, such as fluoromethane $(\approx 6 \%)$, benzoic acid $(\approx 31 \%)$, paracetamol $(\approx 151 \%)$ and coumarin $(219 \%)$.
Finally, no difference in permeation was observed for propane $(\approx-0.6 \%)$.

In order to make comparisons between different molecules easier, the logarithms of base $10, \log \mathrm{P}$, of the permeation coefficients are also presented in table 2 and figure 5 . From figure 5, it can be seen that most permeants have a negative $\log \mathrm{P}$ value, with the most negative corresponding to the four hydrophilic molecules (urea, water, glycine and ammonia). Propane and carbon dioxide, both hydrophobic, have the highest $\log \mathrm{P}$ together with benzoic acid. Fluoromethane, ethanol, isopropanol, phenol, coumarin and paracetamol have $\log \mathrm{P}$ values between -1 and 0.7 . It can also be seen that the majority of the permeants are on the right side of the equality line, indicating a higher $\log \mathrm{P}$ value through the DOPC membrane; only paracetamol and coumarin are on the left of the equality line, indicating higher $\log \mathrm{P}$ value through the mixed DOPC:DOPE bilayer.

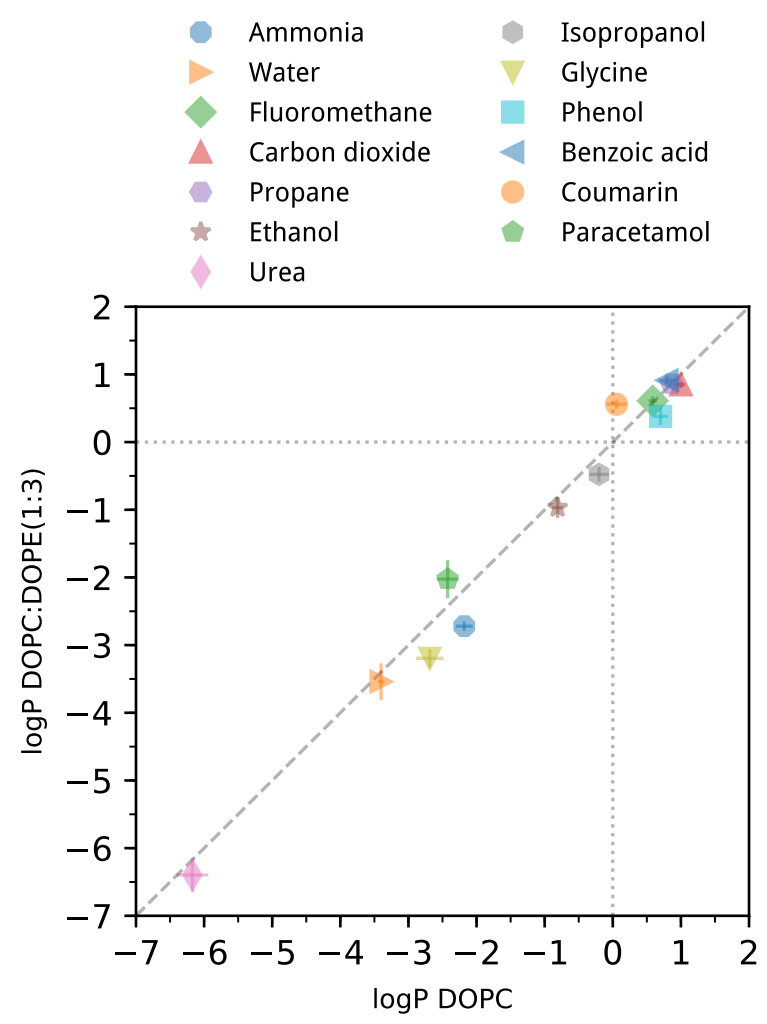

Fig. 5 Comparison of logP values between membranes. In most cases, the standard errors are smaller than the size of the corresponding symbols.

To evaluate whether the membrane composition has a significant effect on the permeability coefficient, we carried out onetailed paired t-tests on the differences in $\log \mathrm{P}$ induced by exposing a permeant to the two different membranes. Two tests were performed on two different permeant groups, one including permeants with molecular weight smaller than $100 \mathrm{~g} \mathrm{~mol}^{-1}$ (smallsized molecules) and one including permeants with molecular weight larger than $100 \mathrm{~g} \mathrm{~mol}^{-1}$ (medium-sized molecules). For molecules with molecular weight lower than $100 \mathrm{~g} \mathrm{~mol}^{-1}$, there is strong evidence $(p-$ value $=0.0019)$ that the $\log \mathrm{P}$ values for the 
Table 2 Permeation coefficients and their logarithm of base 10, for a DOPC and a DOPC:DOPE(1:3) membrane, at T=300 K. Uncertainty is the standard error. An extended table with more lipid compositions is available in the supplementary material. $\alpha$ : MD simulation, $\beta$ : Experimental study.

\begin{tabular}{|c|c|c|c|c|c|}
\hline \multicolumn{3}{|c|}{ This work } & \multicolumn{3}{|c|}{ Previous studies } \\
\hline $\mathrm{P}\left[\mathrm{cms}^{-1}\right]$ & $\log \mathrm{P}$ & Membrane & $\mathrm{P}\left[\mathrm{cms}^{-1}\right]$ & $\log \mathrm{P}$ & Membrane \\
\hline \multicolumn{6}{|l|}{ Urea } \\
\hline$(6.74 \pm 3.03) \times 10^{-7}$ & $-6.17 \pm 0.23$ & DOPC & $5.37 \times 10^{-7}$ & -6.27 & $\mathrm{DMPC}_{298 \mathrm{~K}} \alpha, 130$ \\
\hline$(4.01 \pm 1.95) \times 10^{-7}$ & $-6.40 \pm 0.25$ & DOPC:DOPE(1:3) & $1.41 \times 10^{-6}$ & -5.85 & $\mathrm{DOPC}_{303 \mathrm{~K}} \beta, 53$ \\
\hline \multicolumn{6}{|l|}{ Water } \\
\hline$(3.96 \pm 0.39) \times 10^{-4}$ & $-3.40 \pm 0.05$ & DOPC & $1.22 \times 10^{-2}$ & -1.91 & $\mathrm{DOPC}_{298 \mathrm{~K}} \beta, 56$ \\
\hline \multirow[t]{12}{*}{$(2.89 \pm 1.50) \times 10^{-4}$} & $-3.54 \pm 0.27$ & DOPC:DOPE(1:3) & $7.40 \times 10^{-3}$ & -2.13 & DOPC:DOPE $298 \mathrm{~K} /, 56$ \\
\hline & & & $2.30 \times 10^{-4}$ & -3.64 & $\mathrm{DOPC}_{293 \mathrm{~K}} \beta, 134$ \\
\hline & & & $4.26 \times 10^{-3}$ & -2.37 & $\mathrm{DOPC}_{294 \mathrm{~K}} \beta, 135$ \\
\hline & & & $1.50 \times 10^{-2}$ & -1.82 & $\mathrm{DOPC}_{303 \mathrm{~K}} \beta, 53$ \\
\hline & & & $1.58 \times 10^{-2}$ & -1.80 & $\mathrm{DOPC}_{303 \mathrm{~K}} \alpha, 59$ \\
\hline & & & $1.36 \times 10^{-2}$ & -1.87 & $\mathrm{POPC}_{298 \mathrm{~K}} \beta, 136$ \\
\hline & & & $1.30 \times 10^{-2}$ & -1.89 & $\mathrm{POPC}_{303 \mathrm{~K}} \alpha, 59$ \\
\hline & & & $6.47 \times 10^{-3}$ & -2.19 & $\mathrm{POPC}_{308 \mathrm{~K}}{ }^{\alpha, 85}$ \\
\hline & & & $5.20 \times 10^{-4}$ & -3.28 & $\mathrm{DMPC}_{343 \mathrm{~K}} \beta, 60$ \\
\hline & & & $2.30 \times 10^{-6}$ & -5.64 & $\mathrm{DMPE}_{343 \mathrm{~K}} \beta, 60$ \\
\hline & & & $3.00 \times 10^{-4}$ & -3.52 & $\mathrm{DPPC}_{343 \mathrm{~K}} \beta, 60$ \\
\hline & & & $3.70 \times 10^{-6}$ & -5.43 & $\mathrm{DPPE}_{343 \mathrm{~K}} \beta, 60$ \\
\hline \multicolumn{6}{|l|}{ Glycine } \\
\hline$(2.05 \pm 0.80) \times 10^{-3}$ & $-2.69 \pm 0.20$ & DOPC & $2.00 \times 10^{-11}$ & -10.70 & $\mathrm{DMPC}^{\beta, 137}$ \\
\hline$(6.38 \pm 1.67) \times 10^{-4}$ & $-3.20 \pm 0.14$ & DOPC:DOPE(1:3) & & & \\
\hline \multicolumn{6}{|l|}{ Paracetamol } \\
\hline$(3.76 \pm 1.17) \times 10^{-3}$ & $-2.42 \pm 0.16$ & DOPC & & & \\
\hline$(9.44 \pm 5.10) \times 10^{-3}$ & $-2.03 \pm 0.28$ & DOPC:DOPE(1:3) & & & \\
\hline \multicolumn{6}{|l|}{ Ammonia } \\
\hline$(6.58 \pm 1.57) \times 10^{-3}$ & $-2.18 \pm 0.12$ & DOPC & $1.30 \times 10^{-1}$ & -0.89 & $\mathrm{POPC}_{300 \mathrm{~K}}{ }^{\alpha, 62}$ \\
\hline \multirow[t]{2}{*}{$(1.91 \pm 0.26) \times 10^{-3}$} & $-2.72 \pm 0.07$ & DOPC:DOPE(1:3) & $1.70 \times 10^{-2}$ & -1.77 & $\mathrm{POPE}_{300 \mathrm{~K}} \alpha, 62$ \\
\hline & & & $1.30 \times 10^{-1}$ & -0.89 & $\mathrm{DOPC}_{300 \mathrm{~K}} \alpha, 48$ \\
\hline \multicolumn{6}{|l|}{ Ethanol } \\
\hline$(1.55 \pm 0.24) \times 10^{-1}$ & $-0.81 \pm 0.08$ & DOPC & 2.00 & 0.30 & $\mathrm{POPC}_{308 \mathrm{~K}} \alpha, 84$ \\
\hline \multirow[t]{2}{*}{$(1.08 \pm 0.32) \times 10^{-1}$} & $-0.97 \pm 0.15$ & DOPC:DOPE(1:3) & $8.50 \times 10^{-2}$ & -1.07 & $\mathrm{POPC}_{323 \mathrm{~K}} \alpha, 138$ \\
\hline & & & $3.80 \times 10^{-5}$ & -4.42 & $\mathrm{SOPC}_{298 \mathrm{~K}} \beta, 139$ \\
\hline \multicolumn{6}{|l|}{ Isopropanol } \\
\hline$(6.27 \pm 1.75) \times 10^{-1}$ & $-0.20 \pm 0.15$ & DOPC & & & \\
\hline$(3.34 \pm 0.68) \times 10^{-1}$ & $-0.48 \pm 0.11$ & DOPC:DOPE(1:3) & & & \\
\hline \multicolumn{6}{|l|}{ Coumarin } \\
\hline $1.14 \pm 0.31$ & $0.06 \pm 0.14$ & DOPC & & & \\
\hline $3.62 \pm 0.48$ & $0.56 \pm 0.07$ & DOPC:DOPE(1:3) & & & \\
\hline \multicolumn{6}{|l|}{ Fluoromethane } \\
\hline $3.86 \pm 0.45$ & $0.59 \pm 0.06$ & DOPC & & & \\
\hline $4.07 \pm 0.53$ & $0.61 \pm 0.07$ & DOPC:DOPE(1:3) & & & \\
\hline \multicolumn{6}{|l|}{ Phenol } \\
\hline $5.03 \pm 1.04$ & $0.70 \pm 0.11$ & DOPC & & & \\
\hline $2.40 \pm 0.59$ & $0.38 \pm 0.13$ & DOPC:DOPE(1:3) & & & \\
\hline \multicolumn{6}{|l|}{ Benzoic Acid } \\
\hline $6.27 \pm 1.57$ & $0.80 \pm 0.13$ & DOPC & 2.82 & 0.45 & $\mathrm{DMPC}_{298 \mathrm{~K}} \alpha, 130$ \\
\hline \multirow[t]{3}{*}{$8.19 \pm 0.85$} & $0.91 \pm 0.05$ & DOPC:DOPE(1:3) & $4.40 \times 10^{-5}$ & -4.36 & $\mathrm{DOPC}^{\beta, 140}$ \\
\hline & & & $1.20 \times 10^{-7}$ & -6.92 & $\mathrm{DOPC}_{298 \mathrm{~K}} \beta, 141$ \\
\hline & & & $1.11 \times 10^{-6}$ & -5.95 & $\mathrm{DOPE}_{298 \mathrm{~K}} \beta, 141$ \\
\hline \multicolumn{6}{|l|}{ Propane } \\
\hline $7.33 \pm 1.49$ & $0.86 \pm 0.11$ & DOPC & & & \\
\hline $7.28 \pm 0.86$ & $0.86 \pm 0.06$ & DOPC:DOPE(1:3) & & & \\
\hline Carbon dioxide & & & & & \\
\hline $10.00 \pm 1.2$ & $1.00 \pm 0.06$ & DOPC & 3.00 & 0.48 & POPC:POPE ${ }_{300 \mathrm{~K}}^{\alpha, 62}$ \\
\hline $7.02 \pm 0.56$ & $0.85 \pm 0.04$ & DOPC:DOPE(1:3) & & & \\
\hline
\end{tabular}


mixed DOPC-DOPE bilayer are significantly lower than the corresponding values for the DOPC bilayer. For the three drugs with molecular weight higher than $100 \mathrm{~g} \mathrm{~mol}^{-1}$, there is some evidence $(p-$ value $=0.0496)$ that the $\log \mathrm{P}$ values are instead significantly higher for the mixed DOPC-DOPE bilayer compared to the corresponding values for the DOPC bilayer. Calculation details of the statistical tests conducted can be found in the supplementary material.

Overall, our results are consistent with available experimental and computational data previously reported in the literature.

In particular, Jansen and Blume ${ }^{60}$ examined water permeation through several large unilamellar lipid vesicles. At $\mathrm{T}=343 \mathrm{~K}$, DMPE and DPPE reduced permeation by $\approx 99 \%$ in comparison to pure DMPC and DPPC, respectively. The same behaviour was observed in our study, although the relative difference between the DOPC and DOPC:DOPE membrane was smaller (-27\%). Also Huster et al. ${ }^{56}$ studied water permeation, with $\mathrm{O}^{17}$ nuclear magnetic resonance, through a pure DOPC and a DOPC:DOPE(1:1) bilayer, at $\mathrm{T}=303 \mathrm{~K}$. They reported that for the latter, the permeation coefficient reduced by $\approx 39 \%$, consistent with the present work. Seo et al. ${ }^{141}$ performed a study on the effect of lipid composition on the passive permeation of molecules through PAMPA assays, and observed an $825 \%$ increase in the permeation of benzoic acid through a DOPE phase, in comparison to pure DOPC. Although the PAMPA system differs from the bilayers in our MD simulations, in that the lipid phase in PAMPA is much thicker $(\approx 100 \mu \mathrm{m})$, it is noteworthy that we predicted the same qualitative effect, i.e., a permeation increase in the DOPC:DOPE mixed bilayer compared to pure DOPC (specifically, we observed a $31 \%$ increase). Hub et al. ${ }^{62}$ examined the permeation of ammonia through a pure POPC and a pure POPE bilayer at $\mathrm{T}=300 \mathrm{~K}$. They reported an $\approx 87 \%$ reduction in permeation for the latter, which is consistent with our observation of a $71 \%$ reduction for DOPC:DOPE(1:3). Wennberg et al. ${ }^{69}$ examined the partitioning of ammonia, ethanol and propane in a pure POPC and pure POPE bilayer and they reported that the transfer free energy through the latter was increased for all solutes. Finally, Zocher et al. ${ }^{48}$ also observed a decrease in the permeation of ammonia through a pure DOPE membrane in comparison to a pure DOPC.

Overall, our results are mostly in agreement with the permeation and $\log \mathrm{P}$ values previously reported in the literature. Where permeation coefficients were not available, free energy profiles from the literature can be used to assess the findings. Comparisons of our $\log \mathrm{P}$ values to literature values for each permeant are detailed in the following paragraphs.

Urea. The computed $\log P$ value of -6.17 for the DOPC membrane is close to the -5.85 for $\mathrm{DOPC}^{53}$ at $303 \mathrm{~K}$ and -6.27 for $\mathrm{DMPC}^{130}$ at $298 \mathrm{~K}$ found in the literature.

Water. Studies of water permeation have reported a plethora of results spread over a large range, depending on the experimental and simulation protocols and lipid compositions. In general, $\log \mathrm{P}$ values of water are between -1.15 to -5.64 and our $\log \mathrm{P}$ values of -3.40 for DOPC and -3.54 for DOPE belong to the lower end of this range. In particular, values of this study are two orders of magnitude smaller than the DOPC and POPC permeation coefficients of Mathai et al. ${ }^{59}$, Paula et al. ${ }^{53}$, Huster et al. ${ }^{56}$ and
Koenig et al. ${ }^{136}$, one order of magnitude smaller than those reported by Olbrich et al. ${ }^{135}$ and Comer et al. ${ }^{85}$ and very close to the values of DOPC from Carruthers et al. ${ }^{134}$ and DMPC/DPPC of Jansen and Blume. ${ }^{60}$

Glycine. Chakrabarti et al. ${ }^{137}$ reported a $\log \mathrm{P}$ of -10.7 through large unilamellar vesicles of DMPC, which is somewhat smaller than our result for DOPC.

Ammonia. Our MD simulations underestimated the permeation coefficients of ammonia in comparison to the rest of the literature. With regards to studies of similar lipid compositions, the values presented here are 1 to 2 orders of magnitude smaller than the POPC, POPE and DOPC MD simulations of Hub et al. ${ }^{62}$ and Zocher et al. ${ }^{48}$ The discrepancy with both studies may be ascribed to the different force fields used; specifically, the referenced studies employed the Berger united-atom model for lipids, OPLS for ammonia and TIP4P for water, whereas we used allatom CHARMM, CGenFF and TIP3P, respectively (as detailed in the Simulation Protocol section).

Ethanol. Literature values vary over 6 orders of magnitude and they are mostly negative, apart from the simulation work of Comer et al. ${ }^{84}$ that reported a $\log \mathrm{P}$ value of 0.30 for POPC at $\mathrm{T}=308 \mathrm{~K}$. Ghaemi et al. ${ }^{138}$ computed a $\log \mathrm{P}$ of -1.07 for POPC at $\mathrm{T}=323 \mathrm{~K}$ which is very similar to our values. The experimental work of Ly and Longo ${ }^{139}$, with SOPC, returned a $\log$ P of -4.42 at room temperature. Further validation results were available from the works of MacCallum et al. ${ }^{50}$ and Carpenter et al. ${ }^{142}$ that studied the free energy of ethanol permeating through a DOPC bilayer. In both cases, our findings are almost identical to theirs, qualitatively and quantitatively. Therefore it is expected that also the permeation coefficients would be very similar.

Benzoic Acid. Our results are in good agreement with the simulation work of Lee et al. ${ }^{130}$ who computed a $\log \mathrm{P}$ of 0.45 for DMPC at room temperature, similar to our value of 0.80 for DOPC. These coefficients are both many orders of magnitude higher than the experimental findings for DOPC ${ }^{140,141}$ and DOPE ${ }^{141}$.

Carbon dioxide. The $\log \mathrm{P}$ obtained in our work is very similar to the one by Hub et al. ${ }^{62}$, also from MD simulations, for carbon dioxide permeation through a POPC:POPE bilayer.

Propane. While no reported permeation coefficients were found for propane, MacCallum et al. ${ }^{50}$ have presented the free energy profile of permeating propane. As with ethanol, our findings are in excellent agreement with theirs, observing the same $-3.2 \mathrm{kcal} \mathrm{mol}^{-1}$ free energy trough in the bilayer centre and overall qualitative behaviour. Therefore, it is expected that the permeation coefficient would also be identical.

Isopropanol, fluoromethane. No computational or experimental results were found regarding the permeation of isopropanol or fluoromethane. Ours appears to be the first report of the permeation coefficients for these two molecules.

Phenol, paracetamol and coumarin. While there are no literature permeation studies with similar lipid compositions, Paloncỳovà et al. ${ }^{143}$ have reported free energy profiles of coumarin in a DOPC bilayer at $310 \mathrm{~K}$ that are qualitatively close to our findings, with a global minimum around $1.4 \mathrm{~nm}$ and an energy barrier in the centre of the bilayer. However, quantitatively the results differ considerably, possibly because of the difference 
in temperature and force field, as Paloncỳovà et al. ${ }^{143}$ used the united atom Berger model for lipids and custom parameters for coumarin based on GROMOS 53a6. In our study, the global minimum is $-1.5 \mathrm{kcal} \mathrm{mol}^{-1}$, while they report a global minimum of $-5.7 \mathrm{kcal} \mathrm{mol}^{-1}$ to $-6.7 \mathrm{kcal} \mathrm{mol}^{-1}$. Likewise, in the bilayer centre they report a $-3.5 \mathrm{kcal} \mathrm{mol}^{-1}$ to $-4.1 \mathrm{kcal} \mathrm{mol}^{-1}$ negative peak in contrast to the positive $1.2 \mathrm{kcal} \mathrm{mol}^{-1}$ barrier that was found in this work. Therefore, the permeation coefficient for coumarin from Paloncỳovà et al. ${ }^{143}$ would be probably a few orders of magnitude higher than the one reported here.

\subsection{Hydrogen bonds}

The average number of hydrogen bonds per frame (h.b.p.f.) formed by the permeants is shown in the last column of figure 4. In particular, the total number of hydrogen bonds are shown together with their decomposition into the number of hydrogen bonds formed between the permeant and either the bilayer or the solvent (water). Hydrogen bonds with the solvent are predominant in comparison to the ones formed with the bilayer, and generally increase with the distance from the bilayer centre as also observed in previous studies ${ }^{79,127}$. It is noteworthy however, that hydrophilic and amphiphilic molecules form hydrogen bonds with water almost right down to the bilayer core, showing the propensity of permeants to retain their hydration shell even deep into the hydrophobic region ${ }^{144}$. Quantitatively, as expected, hydrophilic molecules formed more hydrogen bonds with water compared to hydrophobic molecules. Hydrogen bonds with the bilayer can be split in two profile categories. For fluoromethane, carbon dioxide, coumarin and ammonia/DOPC, they are very close to zero along the bilayer. For the rest, hydrogen bonds start to form around $0.1 \mathrm{~nm}$ to $0.5 \mathrm{~nm}$, then reach a maximum at $1.5 \mathrm{~nm}$ to $2.0 \mathrm{~nm}$ (of 0.4 h.b.p.f. for water, urea, glycine and paracetamol, of 0.2 for ethanol, isopropanol, phenol and benzoic acid, of 0.1 for ammonia/DOPC:DOPE) and then fade back to zero in the solvent region.

Overall, no significant difference was observed in the hydrogen bond formations of the molecules with the bilayer between the DOPC and DOPC:DOPE(1:3) membranes, i.e., the lipid composition did not affect the total number of hydrogen bonds formed between the permeants and their environment. Such a finding may appear counterintuitive, due to the presence of an extra hydrogen bond donor in DOPE (part of the headgroup amine moiety) compared to DOPC; however, as was observed in the past ${ }^{11}$, any corresponding additional DOPE hydrogen bonds are mostly formed with either other DOPE or DOPC headgroups, or with solvent molecules, so that permeants are excluded.

\section{Conclusions}

In this work we used atomistic molecular dynamics simulations to examine how changes in the lipid composition can affect the biologically crucial process of passive permeation. The free energy, local diffusion and local resistance profiles, as well as the permeation coefficients were reported for thirteen small molecules and drugs. For eleven of the molecules selected, permeation coefficients were reported for the first time in relation to DOPC and DOPC:DOPE(1:3) membranes. Our key findings are that the presence of the nonlamellar DOPE lipids reduces permeation for the smaller molecules (molecular weight $<100 \mathrm{~g} \mathrm{~mol}^{-1}$ ) while enhances permeation for the largest (molecular weight $>$ $100 \mathrm{~g} \mathrm{~mol}^{-1}$ ). This is the first systematic investigation of the effect of changes in the lamellar vs. nonlamellar lipid composition on membrane permeation. In general, our study represents an advancement towards the development of more realistic membrane models for the in silico prediction of molecular permeation.

\section{Conflicts of interest}

There are no conflicts to declare.

\section{Acknowledgement}

We acknowledge computational support from the UK High-End Computing Consortium for Biomolecular Simulation, HECBioSim (http://hecbiosim.ac.uk), supported by EPSRC (grant no. EP/L000253/1) for the time granted on the ARCHER UK National Supercomputing Service (http://www.archer.ac.uk).

\section{References}

1 G. Cevc and D. Marsh, Phospholipid Bilayers - Physical Principles and Models, John Wiley \& Sons, New York, 1st edn., 1987.

2 O. G. Mouritsen, Life - As a Matter of Fat. The Emerging Science of Lipidomics, Springer, Berlin, 1st edn., 2005.

3 S. M. Bezrukov, Curr. Opin. Colloid Interface Sci., 2000, 5, 237-243.

4 R. Lipowsky and E. Sackmann, Structure and Dynamics of Membranes, Elsevier, Amsterdam, 1995.

5 R. B. Gennis, Biomembranes: Molecular Structure and Function, Springer, Berlin, 1st edn., 1989.

6 K. Gawrisch, V. A. Parsegian, D. A. Hajduk, M. W. Tate, S. M. Graner, N. L. Fuller and R. P. Rand, Biochemistry, 1992, 31, 2856-2864.

7 R. H. Templer, S. J. Castle, A. R. Curran, G. Rumbles and D. R. Klug, Faraday Discuss., 1998, 41-53.

8 G. S. Attard, R. H. Templer, W. S. Smith, A. N. Hunt and S. Jackowski, Proc. Natl. Acad. Sci. U.S.A., 2000, 97, 90329036.

9 R. S. Cantor, Biophys. J., 1999, 76, 2625-2639.

10 S. I. Mukhin and S. Baoukina, Phys. Rev. E, 2005, 71, 061918.

11 A. H. de Vries, A. E. Mark and S. J. Marrink, J. Phys. Chem. $B, 2004,108,2454-2463$.

12 L. Lu and G. A. Voth, J. Phys. Chem. B, 2009, 113, 15011510.

13 M. Orsi and J. W. Essex, PLoS One, 2011, 6,

14 H. Saito, M. Iwayama, H. Takagi, M. Nishimura, T. Miyakawa, K. Kawaguchi, M. Takasu, T. Mizukami and H. Nagao, Int. J. Quantum Chem., 2012, 112, 3834-3839.

15 A. Arouri, K. E. Lauritsen, H. L. Nielsen and O. G. Mouritsen, Chem. Phys. Lipids, 2016, 200, 139-146.

16 S. Ollila, M. T. Hyvönen and I. Vattulainen, J. Phys. Chem. B, 2007, 111, 3139-3150. 
17 M. Orsi, W. Sanderson and J. W. Essex, in Molecular Interactions - Bringing Chemistry to Life, ed. M. G. Hicks and C. Kettner, Beilstein-Institut, Frankfurt, Germany, 2007, pp. 85205.

18 M. Patra, Eur. Biophys. J., 2005, 35, 79-88.

19 M. Carrillo-Tripp and S. E. Feller, Biochemistry, 2005, 44, 10164-10169.

20 H. Binder and K. Gawrisch, J. Phys. Chem. B, 2001, 105, 12378-12390.

21 W. Ding, M. Palaiokostas, W. Wang and M. Orsi, J. Phys. Chem. B, 2015, 119, 15263-15274.

22 W. Ding, M. Palaiokostas, G. Shahane, W. Wang and M. Orsi, J. Phys. Chem. B, 2017, 121, 9597-9606.

23 D. Marsh, Biophys. J., 2007, 93, 3884-3899.

24 L. Yang, L. Ding and H. W. Huang, Biochemistry, 2003, 42, 6631-6635.

25 G. C. Shearman, O. Ces, R. H. Templer and J. M. Seddon, J. Phys. Condens. Matter, 2006, 18, S1105-24.

26 T. Kamo, M. Nakano, Y. Kuroda and T. Handa, J. Phys. Chem. B, 2006, 110, 24987-24992.

27 K. Charalambous, D. Miller, P. Curnow and P. J. Booth, BMC Biochem., 2008, 9, 1-12.

28 P. Curnow, M. Lorch, K. Charalambous and P. J. Booth, J. Mol. Biol., 2004, 343, 213-222.

29 R. S. Cantor, Biochemistry, 1997, 36, 2339-2344.

30 R. S. Cantor, Toxicol. Lett., 1998, 100-101, 451-458.

31 B. Fábián, M. Sega, V. P. Voloshin, N. N. Medvedev and P. Jedlovszky, J. Phys. Chem. B, 2017, 121, 2814-2824.

32 J. T. Mohr, G. W. Gribble, S. S. Lin, R. G. Eckenhoff and R. S. Cantor, J. Med. Chem., 2005, 48, 4172-4176.

33 R. S. Cantor, J. Phys. Chem. B, 1997, 101, 1723-1725.

34 S. Ollila, T. Róg, M. Karttunen and I. Vattulainen, J. Struct. Biol., 2007, 159, 311-323.

35 J. Gullingsrud, A. Babakhani and J. A. McCammon, Mol. Simul., 2006, 32, 831-838.

36 E. Van Den Brink-Van Der Laan, J. Antoinette Killian and B. De Kruijff, Biochimica et Biophysica Acta - Biomembranes, 2004, 1666, 275-288.

37 J. Gullingsrud and K. Schulten, Biophys. J., 2004, 86, 34963509.

38 R. S. Cantor, Biophys. J., 1999, 77, 2643-2647.

39 A. K. Mandagere, T. N. Thompson and K.-K. Hwang, J. Med. Chem., 2002, 45, 304-311.

$40 \mathrm{M}$. Orsi and J. W. Essex, in Molecular simulations and biomembranes: from biophysics to function, ed. P. C. Biggin and M. S. P. Sansom, RSC Publishing, Cambridge, 2010, pp. 76-90.

41 A. Finkelstein, Water movement through lipid bilayers, pores, and plasma membranes: theory and reality, Wiley, 1987.

42 G. Camenisch, G. Folkers and H. van de Waterbeemd, Pharm. Acta Helv., 1996, 71, 309-327.

43 T. X. Xiang and B. D. Anderson, J. Membr. Biol., 1994, 140, 111-122.
44 T. X. Xiang and B. D. Anderson, Biophys. J., 1998, 75, 26582671.

45 S. J. Marrink and H. J. C. Berendsen, J. Phys. Chem., 1996, 100, 16729-16738.

46 D. Bemporad, J. W. Essex and C. Luttmann, J. Phys. Chem. $B, 2004,108$, 4875-4884.

47 D. Bemporad, C. Luttmann and J. W. Essex, Biophys. J., 2004, 87, 1-13.

48 F. Zocher, D. Van Der Spoel, P. Pohl and J. S. Hub, Biophys. J., 2013, 105, 2760-2770.

49 J. M. Nitsche and G. B. Kasting, J. Pharm. Sci., 2013, 102, 2005-2032.

50 J. L. MacCallum, W. F. D. Bennett and D. P. Tieleman, Biophys. J., 2008, 94, 3393-3404.

51 M. Nakamura and T. Osakai, J. Electroanal. Chem., 2016, 779, 55-60.

52 B. A. Lewis and D. M. Engelman, J. Mol. Biol., 1983, 166, 211-217.

53 S. Paula, A. G. Volkov, A. N. Van Hoek, T. H. Haines and D. W. Deamer, Biophys. J., 1996, 70, 339-348.

54 T. Sugii, S. Takagi and Y. Matsumoto, J. Chem. Phys., 2005, 123, 184714.

55 M. H. Lee, B. Lim, J. W. Kim, E. J. An and D. Lee, Soft Matter, 2012, 8, 1539.

56 D. Huster, a. J. Jin, K. Arnold and K. Gawrisch, Biophys. J., 1997, 73, 855-864.

57 D. R. Carl and S. E. Feller, Langmuir, 2003, 19, 8560-8564.

58 A. L. Rabinovich, N. K. Balabaev, M. G. Alinchenko, V. P. Voloshin, N. N. Medvedev and P. Jedlovszky, J. Chem. Phys., 2005, 122, 84906.

59 J. C. Mathai, S. Tristram-Nagle, J. F. Nagle and M. L. Zeidel, J. Gen. Physiol., 2008, 131, 69-76.

60 M. Jansen and A. Blume, Biophys. J., 1995, 68, 997-1008.

61 A. C. V. Johansson and E. Lindahl, J. Chem. Phys., 2009, 130, 185101.

62 J. S. Hub, F. K. Winkler, M. Merrick and B. L. De Groot, J. Am. Chem. Soc., 2010, 132, 13251-13263.

63 H. Saito and W. Shinoda, J. Phys. Chem. B, 2011, 115, 15241-15250.

64 B. B. Issack and G. H. Peslherbe, J. Phys. Chem. B, 2015, 119, 150213162609001.

65 W. K. Subczynski, J. S. Hyde and A. Kusumi, Proc. Natl. Acad. Sci. U. S. A., 1989, 86, 4474-4478.

66 S. J. McKinnon, S. L. Whittenburg and B. Brooks, J. Phys. Chem., 1992, 96, 10497-10506.

67 J. Widomska, M. Raguz and W. K. Subczynski, Biochim. Biophys. Acta, 2007, 1768, 2635-2645.

68 P. Jedlovszky and M. Mezei, J. Phys. Chem. B, 2003, 107, 5322-5332.

69 C. L. Wennberg, D. van der Spoel and J. S. Hub, J. Am. Chem. Soc., 2012, 134, 5351-5361.

70 S. Purushothaman, J. Cama and U. F. Keyser, Soft Matter, 2016, 12, 2135-2144. 
71 M. Orsi and J. W. Essex, Faraday Discuss., 2013, 249-272.

72 H. Meyer, Archiv f. experiment. Pathol. u. Pharmakol, 1899, 42, 109-118.

73 C. E. Overton, Studien über die Narkose zugleich ein Beitrag zur Allgemeinen Pharmakologie, Fischer, 1901.

74 A. Finkelstein, J. Gen. Physiol., 1976, 68, 127-135.

75 J. M. Diamond and Y. Katz, J. Membrain Biol., 1974, 17, 121-154.

76 H. Berendsen and S. J. Marrink, J. Macromol. Sci. Part A Pure Appl. Chem., 1993, 65, 2513-2520.

77 S.-J. Marrink and H. J. C. Berendsen, J. Phys. Chem., 1994, 98, 4155-4168.

78 E. Awoonor-Williams and C. N. Rowley, Biochimica et Biophysica Acta (BBA) - Biomembranes, 2016, 1858, 16721687.

79 W. Shinoda, Biochim. Biophys. Acta, 2016, 1858, 22542265.

80 B. L. Lee, K. Kuczera, C. R. Middaugh and G. S. Jas, J. Chem. Phys., 2016, 144, 245103.

81 G. M. Torrie and J. P. Valleau, J. Comput. Phys., 1977, 23, 187-199.

82 S. Kumar, J. M. Rosenberg, D. Bouzida, R. H. Swendsen and P. A. Kollman, J. Comput. Chem., 1992, 13, 1011-1021.

83 S. Kumar, J. M. Rosenberg, D. Bouzida, R. H. Swendsen and P. A. Kollman, J. Comput. Chem., 1995, 16, 1339-1350.

84 J. Comer, K. Schulten and C. Chipot, J. Chem. Theory Comput., 2014, 10, 2710-2718.

85 J. Comer, K. Schulten and C. Chipot, J. Chem. Theory Comput., 2014, 10, 554-564.

86 J. Comer, C. Chipot and F. D. González-Nilo, J. Chem. Theory Comput., 2013, 9, 876-882.

87 K. Gaalswyk, E. Awoonor-Williams and C. N. Rowley, J. Chem. Theory Comput., 2016, 12, 5609-5619.

88 G. Hummer, New J. Phys., 7, 34.

89 T. B. Woolf and B. Roux, J. Am. Chem. Soc., 1994, 116, 5916-5926.

90 B. J. Berne, M. Borkovec and J. E. Straub, J. Phys. Chem., 1988, 92, 3711-3725.

91 P. L. O'neill, D. Nicolaides, D. Honnery, J. Soria and Others, 15th Australasian Fluid Mechanics Conference The University of Sydney, Sydney, Australia, 2004, pp. 13-17.

92 M. Jansen and A. Blume, Biophys. J., 1995, 68, 997-1008.

93 K. Huang and A. E. García, Biophysical Journal, 2013, 104, $412-420$.

94 G. Grasso, S. Muscat, M. Rebella, U. Morbiducci, A. Audenino, A. Danani and M. A. Deriu, Journal of Biomechanics, 2018, 73, 137 - 144.

95 S. Paula, A. Volkov, A. V. Hoek, T. Haines and D. Deamer, Biophysical Journal, 1996, 70, 339 - 348.

96 C. Chipot and J. Comer, Scientific Reports, 2016, 6, 35913.

97 N. J. Yang and M. J. Hinner, in Getting Across the Cell Membrane: An Overview for Small Molecules, Peptides, and Proteins, ed. A. Gautier and M. J. Hinner, Springer New York,
New York, NY, 2015, pp. 29-53.

98 M. J. Abraham, T. Murtola, R. Schulz, S. Páll, J. C. Smith, B. Hess and E. Lindahl, SoftwareX, 2015, 1-2, 19-25.

99 S. Pronk, S. Páll, R. Schulz, P. Larsson, P. Bjelkmar, R. Apostolov, M. R. Shirts, J. C. Smith, P. M. Kasson, D. van der Spoel, B. Hess and E. Lindahl, Bioinformatics, 2013, 29, 845-854.

100 B. Hess, C. Kutzner, D. v. der Spoel and E. Lindahl, J. Chem. Theory Comput., 2008, 4, 435-447.

101 D. Van Der Spoel, E. Lindahl, B. Hess, G. Groenhof, A. E. Mark and H. J. C. Berendsen, J. Comput. Chem., 2005, 26, 1701-1718.

102 E. Lindahl, B. Hess and D. van der Spoel, J. Mol. Model., 2001, 7, 306-317.

103 H. J. C. Berendsen, D. van der Spoel and R. van Drunen, Comput. Phys. Commun., 1995, 91, 43-56.

104 U. Essmann, L. Perera, M. L. Berkowitz, T. Darden, H. Lee and L. G. Pedersen, J. Chem. Phys., 1995, 103, 8577-8593.

105 J. B. Klauda, R. M. Venable, J. A. Freites, J. W. O’Connor, D. J. Tobias, C. Mondragon-Ramirez, I. Vorobyov, A. D. MacKerell and R. W. Pastor, J. Phys. Chem. B, 2010, 114, 7830-7843.

106 R. W. Pastor and A. D. Mackerell, Jr, J. Phys. Chem. Lett., 2011, 2, 1526-1532.

107 K. Vanommeslaeghe, E. Hatcher, C. Acharya, S. Kundu, S. Zhong, J. Shim, E. Darian, O. Guvench, P. Lopes, I. Vorobyov, a. D. Mackerell and A. Manuscript, J. Comput. Chem., 2010, 31, 671-690.

108 W. Yu, X. He, K. Vanommeslaeghe and A. D. MacKerell, Jr, J. Comput. Chem., 2012, 33, 2451-2468.

109 W. L. Jorgensen, J. Chandrasekhar, J. D. Madura, R. W. Impey and M. L. Klein, J. Chem. Phys., 1983, 79, 926.

110 G. Bussi, D. Donadio and M. Parrinello, J. Chem. Phys., 2007, 126, 014101.

111 H. J. C. Berendsen, J. P. M. Postma, W. F. van Gunsteren, A. DiNola and J. R. Haak, J. Chem. Phys., 1984, 81, 3684 3690.

112 M. Parrinello and A. Rahman, Phys. Rev. Lett., 1980, 45, 1196-1199.

113 M. Parrinello and A. Rahman, J. Appl. Phys., 1981, 52, 71827190.

114 M. Parrinello and A. Rahman, J. Chem. Phys., 1982, 76, 2662-2666.

115 M. Orsi, Mol. Phys., 2014, 112, 1566-1576.

116 S. Miyamoto and P. A. Kollman, J. Comput. Chem., 1992, 13, 952-962.

117 B. Hess, H. Bekker, H. J. C. Berendsen and J. G. E. M. Fraaije, J. Comput. Chem., 1997, 18, 1463-1472.

118 B. Hess, J. Chem. Theory Comput., 2008, 4, 116-122.

119 H. Flyvbjerg and H. G. G. Petersen, J. Chem. Phys., 1989, 91, 461.

120 H. J. C. Berendsen, A Student's Guide to Data and Error Analysis, Cambridge University Press, Cambridge, 2011.

121 J. S. Hub, B. L. de Groot and D. v. der Spoel, J. Chem. Theory 
Comput., 2010, 6, 3713-3720.

122 B. Roux, Comput. Phys. Commun., 1995, 91, 275-282.

123 P. Gkeka, P. Angelikopoulos, L. Sarkisov and Z. Cournia, PLOS Computational Biology, 2014, 10, 1-10.

124 G. Grasso, M. A. Deriu, M. Prat, L. Rimondini, E. Verne, A. Follenzi and A. Danani, The Journal of Physical Chemistry $B, 2015,119,8239-8246$.

125 J. L. MacCallum and D. P. Tieleman, J. Am. Chem. Soc., 2006, 128, 125-130.

126 R. W. Tejwani, M. E. Davis, B. D. Anderson and T. R. Stouch, J. Pharm. Sci., 2011, 100, 2136-2146.

127 R. W. Tejwani, M. E. Davis, B. D. Anderson and T. R. Stouch, Mol. Pharm., 2011, 8, 2204-2215.

128 D. Bemporad, C. Luttmann and J. W. Essex, Biochim. Biophys. Acta, 2005, 1718, 1-21.

129 M. Orsi, W. E. Sanderson and J. W. Essex, J. Phys. Chem. B, 2009, 113, 12019-12029.

130 C. T. Lee, J. Comer, C. Herndon, N. Leung, A. Pavlova, R. V. Swift, C. Tung, C. N. Rowley, R. E. Amaro, C. Chipot, Y. Wang and J. C. Gumbart, J. Chem. Inf. Model., 2016, 56, 721-733.

131 C. Neale and R. Pomès, Biochim. Biophys. Acta, 2016, 1858, 2539-2548.

132 M. Orsi, M. G. Noro and J. W. Essex, J. R. Soc. Interface, 2011, 8, 826-841.
133 M. Orsi and J. W. Essex, Soft Matter, 2010, 6, 3797-3808.

134 A. Carruthers and D. L. Melchior, Biochemistry, 1983, 22, 5797-5807.

135 K. Olbrich, W. Rawicz, D. Needham and E. Evans, Biophys. J., 2000, 79, 321-327.

136 S. H. Koenig, Q. F. Ahkong, R. D. Brown, 3rd, M. Lafleur, M. Spiller, E. Unger and C. Tilcock, Magn. Reson. Med., 1992, 23, 275-286.

137 A. C. Chakrabarti and D. W. Deamer, Biochim. Biophys. Acta, 1992, 1111, 171-177.

138 Z. Ghaemi, M. Minozzi, P. Carloni and A. Laio, J. Phys. Chem. $B, 2012,116,8714-8721$.

139 H. V. Ly and M. L. Longo, Biophys. J., 2004, 87, 1013-1033.

140 J. A. Ruell, K. L. Tsinman and A. Avdeef, Eur. J. Pharm. Sci., 2003, 20, 393-402.

141 P. R. Seo, Z. S. Teksin, J. P. Y. Kao and J. E. Polli, Eur. J. Pharm. Sci., 2006, 29, 259-268.

142 T. S. Carpenter, D. A. Kirshner, E. Y. Lau, S. E. Wong, J. P. Nilmeier and F. C. Lightstone, Biophys. J., 2014, 107, 630641.

143 M. Paloncýová, K. Berka and M. Otyepka, J. Chem. Theory Comput., 2012, 8, 1200-1211.

144 A. C. V. Johansson and E. Lindahl, Proteins, 2008, 70, 13321344. 